\title{
Base changes in the fliC gene of Edwardsiella tarda: possible effects on flagellation and motility
}

\author{
Jun Okuda ${ }^{1, *}$, Fumiyasu Murayama ${ }^{1}$, Eisuke Yamanoi $^{1}$, Emi Iwamoto $^{1}$, \\ Satoru Matsuoka ${ }^{2}$, Mitsuaki Nishibuchi ${ }^{3}$, Toshihiro Nakai ${ }^{1}$ \\ ${ }^{1}$ Graduate School of Biosphere Science, Hiroshima University, Higashi-Hiroshima, Hiroshima 739-8528, Japan \\ ${ }^{2}$ Ehime Prefectural Chuyo Fisheries Experimental Station, Iyo, Ehime 799-3125, Japan \\ ${ }^{3}$ Center for Southeast Asian Studies, Kyoto University, Kyoto 606-8501, Japan
}

\begin{abstract}
Edwardsiella tarda is a broad host-range pathogen infecting both animals and humans. E. tarda isolates from red sea bream Pagrus major are non-motile, whereas isolates from Japanese eel Anguilla japonica and Japanese flounder Paralichthys olivaceus are motile with peritrichous flagella. We compared the fliC gene coding for flagellin (FliC) in motile and non-motile E. tarda strains isolated from diseased fish. Twenty-two amino acid residues differed in the predicted FliC amino acid sequences between non-motile and motile strains. There were no significant differences either in the upstream sequences regulating transcription of the fliC gene or in the fliC transcript levels between motile and non-motile strains. The predicted secondary structure of FliC in non-motile E. tarda differed from that of motile strains, and the modeled data suggested that the secondary structure may be the important factor responsible for non-flagellation in the non-motile strains.
\end{abstract}

KEY WORDS: Edwardsiella tarda fliC gene sequence $\cdot$ Base changes $\cdot$ Flagellation $\cdot$ Motility Red sea bream $\cdot$ Japanese flounder

\section{INTRODUCTION}

Edwardsiella tarda, a member of the family Enterobacteriaceae, was first reported as a pathogen associated with the so-called 'red disease' of the Japanese eel Anguilla japonica (Hoshina 1962). Since then, the bacterium has been isolated from humans (Jordan \& Hadley 1969, Clarridge et al. 1980, Wilson \& Waterer 1989, Janda \& Abbott 1993, Mowbray et al. 2003) and other animals including fish (Meyer \& Bullock 1973, Wakabayashi \& Egusa 1973), amphibians (Sharma et al. 1974, Kourany et al. 1977), reptiles (Otis \& Behler 1973, Goldstein et al. 1981), birds (Winsor et al. 1981, Cook \& Tappe 1985), and mammals (Kourany et al. 1977, Van Assche 1991).

Edwardsiella tarda causes systemic infection in a wide variety of cultured seawater and freshwater fish (Plumb 1999, Muroga 2001). The disease itself is called edwardsiellosis, and is a serious problem for the aquaculture industry throughout the world owing to a lack of effective chemotherapeutics and commercially available vaccines. In Japan, the presence of 2 phenotypes, typical and atypical, has long been recognized among E. tarda isolates from diseased fish. Typical strains are motile with peritrichous flagella and produce no acids from arabinose, sucrose, trehalose, or mannitol (Yasunaga et al. 1982, Sakazaki 2001), and have been isolated from the freshwater Japanese eel and the marine Japanese flounder Paralichthys olivaceus (Wakabayashi \& Egusa 1973, Nakatsugawa 1983). In contrast, atypical strains, which are nonmotile and produce acids from arabinose and mannitol but not from trehalose or sucrose, have been isolated from the marine crimson sea bream Evynnis japonicus and red sea bream Pagrus major (Kusuda et al. 1977, Yasunaga et al. 1982). There is no description of the non-motile atypical strains of E. tarda in Bergey's Manual (Sakazaki 2001). Matsuyama et al. (2005) reported a difference in experimental pathogenicity in some marine fish between typical (motile) and atypical (non-motile) strains. The typical strain is highly pathogenic to Japanese flounder but not to red sea bream, 
while the atypical strain exhibits high virulence only in red sea bream.

Bacterial flagella are important structures for pathogenic bacteria because they provide motility and increase adhesion to mucosal surfaces (Ramos et al. 2004). The flagellar filament is composed of approximately 20000 subunits of the protein known as flagellin (FliC). Expression of flagella can be controlled by various genes in response to environmental changes. McIntosh \& Austin (1991) showed that another fish pathogen, Aeromonas salmonicida, expresses flagella only at supra-optimal environmental temperatures (from 30 to $37^{\circ} \mathrm{C}$ ). In Edwardsiella tarda, the fliC gene coding for the FliC protein was previously cloned and sequenced from strain PPD130/91, a motile strain (Tan et al. 2002). The other conditions and genes related to the expression of flagella are unknown in E. tarda.

In this study we determined the mechanism of motility expression and flagellation in typical and atypical strains of Edwardsiella tarda. We show that non-motile strains do not form flagella in vitro in response to changing environmental temperatures. The data suggests that an alteration of the fliC gene sequence in non-motile strains is likely to be responsible for the deficiency in flagella formation in atypical strains.

\section{MATERIALS AND METHODS}

Bacterial strains and media. Thirteen Edwardsiella tarda strains isolated from diseased Japanese flounder or diseased red sea bream were used (Table 1). The E. tarda strains were grown in Triptic-Soy broth (TSB, Eiken) or on Tryptic-Soy agar (TSA, Nissui) at $30^{\circ} \mathrm{C}$ unless otherwise indicated.
Motility tests. The motility of the test strain was examined using a wet mount method with a light microscope (the direct method), and sulfide indole motility (SIM) media (Eiken) (the indirect method) at a variety of temperatures $\left(15,20,25,30,35\right.$, or $\left.40^{\circ} \mathrm{C}\right)$. For the direct wet mount method, the test strains were grown in TSA at $30^{\circ} \mathrm{C}$.

Utilization of carbohydrates. Bacteria were inoculated into bromocresol purple semisolid medium (Eiken) containing each of the 4 carbohydrates L-arabinose, sucrose, trehalose, and D-mannitol at $1 \%$ and incubated at $30^{\circ} \mathrm{C}$ for $2 \mathrm{wk}$.

Flagella staining. Edwardsiella tarda strains were incubated in TSB at $25^{\circ} \mathrm{C}$ for $12 \mathrm{~h}$ and the cells were fixed by addition of $0.5 \%$ neutralized formalin. The bacterial flagella were stained using the NishizawaSugawara method (Shitara 1988).

Analysis of the fliC gene and its upstream sequence. The open reading frame (ORF) of the fliC gene was amplified using PCR with primers 5'-Fla (ATG) and 3'-Fla (stop) (Table 2) that were designed from the reported sequence of a motile strain, PPD130/91 (GenBank Accession No. AF487406) (Tan et al. 2002). PCR amplification was performed using Taq polymerase (Ex Taq; Takara Biochemicals), and the thermal cycling conditions were 30 cycles of denaturation at $95^{\circ} \mathrm{C}$ for $1 \mathrm{~min}$, annealing at $53^{\circ} \mathrm{C}$ for $1 \mathrm{~min}$, and extension at $72^{\circ} \mathrm{C}$ for $1.5 \mathrm{~min}$, followed by a final extension step at $72^{\circ} \mathrm{C}$ for $5 \mathrm{~min}$. The amplified PCR products were cloned into the pGEM-T easy vector (Promega) and sequenced. Using the fliC gene sequence of PPD130/91, we designed 3 primers, 3'-Fla-TSP1, 3'-Fla-TSP2, and 3'-Fla-TSP3 (Table 2). PCR amplification of fragments containing the $199 \mathrm{bp}$ sequence upstream of the fliC ORF was performed

Table 1. Edwardsiella tarda. Motility, flagellation and carbohydrate utilization of 13 strains used in this study. Motility was assessed using a direct wet-mount method, and an indirect test with sulphide indole motility medium. Flagella presence was confirmed using light microscopy

\begin{tabular}{|c|c|c|c|c|c|c|c|c|c|c|}
\hline \multirow[t]{2}{*}{ Strain } & \multicolumn{3}{|c|}{ Isolation } & \multicolumn{2}{|c|}{ Motility -} & \multirow[t]{2}{*}{ Flagella } & \multicolumn{4}{|c|}{$\longrightarrow$ Utilization of } \\
\hline & Source & Place & Year & Direct & Indirect & & L-arabinose & sucrose & trehalose & D-mannitol \\
\hline FK1051 & Japanese flounder & Hiroshima & 2001 & + & + & + & - & - & - & - \\
\hline NUF251 & Japanese flounder & Nagasaki & 1986 & + & + & + & - & - & - & - \\
\hline NUF82 & Japanese flounder & Nagasaki & 1984 & + & + & + & - & - & - & - \\
\hline PE113 & Japanese flounder & Ehime & 2001 & + & + & + & - & - & - & - \\
\hline E01-33 & Japanese flounder & Ehime & 2001 & + & + & + & - & - & - & - \\
\hline E01-37 & Japanese flounder & Ehime & 2001 & + & + & + & - & - & - & - \\
\hline NE8003 & Japanese flounder & Ehime & 1980 & + & + & + & - & - & - & - \\
\hline E01-14 & Red sea bream & Ehime & 2001 & - & - & - & + & - & - & - \\
\hline E01-17 & Red sea bream & Ehime & 2001 & - & - & - & + & - & - & - \\
\hline E01-40 & Red sea bream & Ehime & 2001 & - & - & - & + & - & - & - \\
\hline REF179 & Red sea bream & Mie & 2001 & - & - & - & + & - & - & - \\
\hline REF146 & Red sea bream & Mie & 1996 & - & - & - & + & - & - & - \\
\hline REF0001 & Red sea bream & Mie & 1996 & - & - & - & + & - & - & - \\
\hline
\end{tabular}


Table 2. Edwardsiella tarda. Oligonucleotides used for PCR amplification of the fiC gene in this study

\begin{tabular}{|c|c|c|}
\hline Designation & Nucletide position & Sequence \\
\hline 5'-Fla (ATG) & $200-220^{\mathrm{a}}$ & 5'-ATGGCACAAGTAATTAATACC-3' \\
\hline 5'-Fla (968) & $968-985^{\mathrm{a}}$ & 5'-GGTACTACAGCCGTAACC-3' \\
\hline 3'-Fla (stop) & $1430-1450^{\mathrm{a}}$ & 5'-TTAACGCAGCAGAGACAGGAC-3' \\
\hline 3'-Fla-TSP1 & $282-300^{\mathrm{a}}$ & 5'-GAAGACAGACGCTCGATGG-3' \\
\hline 3'-Fla-TSP2 & $239-261^{\mathrm{a}}$ & 5'-GATTTGTTCAGGTTGGTTCTGTGC-3' \\
\hline 3'-Fla-TSP3 & $218-238^{\mathrm{a}}$ & 5'-CATCAGCGACAGGCTGTTGG-3' \\
\hline 3'-Fla-TSP3-2 & $209-239^{a}$ & 5'-CCATCAGCGACAGGCTGTTGGTATTAATTAC-3' \\
\hline 5'-Fla-1 & $1-21^{\mathrm{a}}$ & 5'-CGATGGGTCAATAGAAGCAGG-3' \\
\hline $5^{\prime}-$ Fla-2 & $1-34^{\mathrm{a}}$ & 5'-CGATGGGCCAATAGAAGCAGGAAAATGGGGGCTT-3 \\
\hline 5'-ET-gyrA & $428-447^{\mathrm{b}}$ & 5'-GATGTTCGGGATGGCCTGAA-3' \\
\hline 3'-ET-gyrA & $709-729^{b}$ & 5'-GCCAACAGCTCATGAGCAATC-3' \\
\hline \multicolumn{3}{|c|}{${ }^{\mathrm{a}}$ Nucleotide positions in Fig. 2} \\
\hline
\end{tabular}

using the 3 primers with a DNA walking kit (DNA Walking SpeedUp Premix Kit, Seegene) according to the manufacturer's protocol. The amplified PCR products were cloned into the pGEM-T easy vector and sequenced. A search for the promoter sequence (-10 and -35 regions) in the 199 bp sequence was performed using the software GENETYX-MAC version 12.2.5 (GENETYX).

lac $Z$ transcriptional fusion assay. A plasmid-borne lac $Z$ transcriptional fusion was constructed by cloning the DNA fragment containing the presumed promoter region (the $199 \mathrm{bp}$ upstream sequence) and the N-terminal coding region of the fliC gene into the lac $Z$ fusion site of the pUJ8 vector (de Lorenzo et al. 1990). Briefly, the $240 \mathrm{bp}$ sequence comprising the $199 \mathrm{bp}$ sequence upstream of the fliC ORF and the $41 \mathrm{bp} 5^{\prime}$ proximal sequence of the fliC ORF was amplified by PCR using primers 5'-Fla-1 and 3'-Fla-TSP3 (for FK1051 and E01-17) or primers 5'-Fla-2 and 3'-Fla-TSP3-2 (for REF146) (Table 2). The amplicons were cloned into the pUJ8 vector, and the resultant plasmids were transformed into Escherichia coli DH5 $\alpha$. The $\beta$-galactosidase assay of the transformants was performed as described previously (Okuda \& Nishibuchi 1998). The $\beta$-galactosidase activities were compared using the Student's $t$-test for significant differences $(p<0.05)$.

fliC transcript analysis by RT-PCR. For reverse transcription (RT)-PCR, bacterial cells were cultured in TSB at $25^{\circ} \mathrm{C}$ overnight. Total RNAs from 13 Edwardsiella tarda strains isolated from diseased Japanese flounder or diseased red sea bream (Table 1) were isolated with ISOGEN (Nippongene) using the method recommended by the manufacturer. The RNAs were purified again after treatment with RNase-free DNase I (Takara), and the amount and purity of the RNAs were determined by measuring $\mathrm{A}_{260}$ and $\mathrm{A}_{280}$. A total of $100 \mathrm{ng}$ of the RNA was used to amplify the fliC and
gyrA transcripts by RT-PCR. The gyrA transcript level was used as the internal control for RT-PCR as described previously (Tanaka et al. 2005). RT-PCR was performed using the SuperScript One-Step RT-PCR with Platinum Taq system (Invitrogen) and primers for fliC (5'-Fla [968] and 3'-Fla [stop] primers) and gyrA (5'-ET-gyrA and 3'-ET-gyrA primers designed from the E. tarda gyrA sequence in GenBank Accession No. DQ019315) (Table 2). The RT-PCR was performed with the following cycle profiles: 30 cycles for fliC annealing at $52^{\circ} \mathrm{C}$, and 30 cycles for gyrA annealing at $50^{\circ} \mathrm{C}$. The RT-PCR products (483 bp for fliC and $302 \mathrm{bp}$ for $g y r A$ ) were visualized using ethidium bromide after $2 \%$ agarose gel electrophoresis. Semiquantitative RT-PCR assay was carried out as described previously (Okuda et al. 2005) with slight modifications. The relative expression level of the fliC transcript was measured by normalizing the PCR product of the fliC gene to that of the gyrA gene. After the densitometric intensity of the RT-PCR products was quantified with NIH Image software, the relative expression level of the fliC transcript was calculated as the ratio of the final RT-PCR product of the fliC gene to that of the gyrA gene (fliC:gyrA ratio). The amount of contaminating chromosomal DNA in each sample was determined in control reactions without reverse transcriptase.

Prediction of FliC secondary structure. We predicted the secondary structure of the FliC using the Chou-Fasman algorithm from the deduced amino acid sequence (Chou \& Fasman 1978) using the software GENETYX-MAC version 12.2.5.

Nucleotide sequence accession numbers. The fliC nucleotide sequences of Edwardsiella tarda FK1051, E01-17, and REF146 were lodged in the DNA Data Bank of Japan (DDBJ) nucleotide sequence database under accession numbers AB271128, AB271129, and AB271130, respectively. 


\section{RESULTS}

\section{Motility, flagellation, and carbohydrate utilization}

All 7 Edwardsiella tarda strains isolated from diseased Japanese flounder showed motility using both the direct and indirect tests, whereas all 6 E. tarda strains isolated from diseased red sea bream did not (Table 1). Photographs of representative strains using the indirect motility test are shown in Fig. 1A. In addition, when the motile and non-motile strains were grown in the SIM medium (indirect test) at a variety of temperatures $\left(15,20,25,30,35\right.$, or $\left.40^{\circ} \mathrm{C}\right)$, the results were the same: motile and non-motile strains were unchanged (data not shown).

Flagella were observed only in the motile strains and not with non-motile strains (Table 1). Photographs of representative strains are shown in Fig. 1B.

None of the 7 Edwardsiella tarda strains isolated from diseased Japanese flounder utilized L-arabinose, sucrose, trehalose, or D-mannitol; in contrast, all 6 E. tarda strains isolated from diseased red sea bream produced acid and gas from arabinose, but not from mannitol, trehalose, or sucrose (Table 1).

(A)

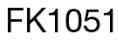

REF146
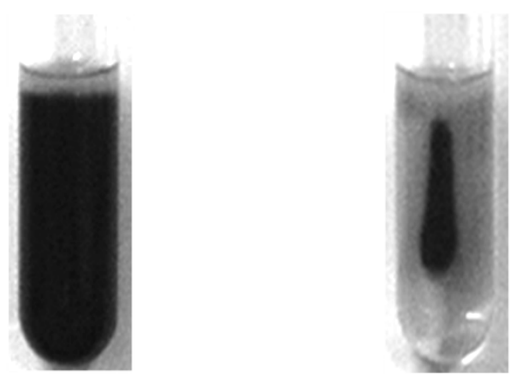

(B)

FK1051

REF146
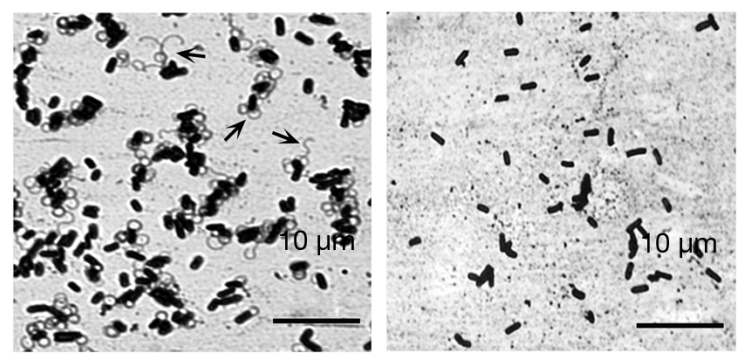

Fig. 1. Edwardsiella tarda. (A) Indirect motility test and (B) direct detection of flagella for motile (FK1051) and non-motile (REF146) strains. Black arrows in (B) indicate representative flagella

\section{Comparison of the flic ORF}

The fliC ORF sequence was successfully amplified from all 6 non-motile strains as well as from the 7 motile strains. The amplified PCR products from FK1051 representing a motile strain and E01-17 and REF146 representing non-motile strains were cloned and sequenced. The fliC ORF sequence of a motile strain, PPD130/91, was obtained from the reported sequence (Tan et al. 2002). Many base differences were detected in the fliC ORFs of the 2 non-motile strains compared with those of the motile strains PPD130/91 and FK1051 (Fig. 2), and all the fliC ORFs were the same length (Fig. 2). The deduced amino acid sequences of the fliC gene cloned from E01-17 and REF146 were compared with those from PPD130/91 and FK1051 (Fig. 3). The amino acid sequence of the 2 motile strains PPD130/91 and FK1051 differed by only 2 residues. The amino acid sequences of the 2 non-motile strains E01-17 and REF146 were identical, and 22 residues differed between motile and nonmotile strains. The influence of these residue differences on the FliC structure was investigated using computer structural modeling software. As shown in Fig. 4, the secondary structure of FliC predicted from the amino acid sequences of the non-motile E01-17 and REF146 (Fig. 4B) is clearly different from that of motile FK1051 (Fig. 4A). The distinct difference in the FliC secondary structure of the non-motile strains compared with that of motile FK1051 is the presence of an additional $\beta$-turn from amino acids 219 to 222 . Owing to the additional $\beta$-turn, the secondary structure of the FliC protein of non-motile strains is an extended form (Fig. 4B), whereas that of motile FK1051 has a hairpin loop structure (Fig. 4A).

\section{Comparison of sequences upstream of the fliC ORF, and flic transcript levels}

We then determined whether the transcription of the fliC gene in non-motile strains was down-regulated because of mutations in the fliC promoter or the

Fig. 2. Edwardsiella tarda. Comparison of strains using alignment of the fliC gene ORF and its $199 \mathrm{bp}$ upstream region. PPD130/91 and FK1051 are motile strains; E01-17 and REF146 are non-motile strains. Sequences showing homology with consensus sequences such as a Shine-Dalgarno sequence (S.D.), and -10 and -35 regions of the promoter are indicated by dots above sequences. The ATG and TTA in bold at nucleotide positions 200 to 202 and 1448 to 1450 are the start and stop codons of the fliC gene, respectively. Identical nucleotides are indicated by dashes 
1

$-35 \quad 50$

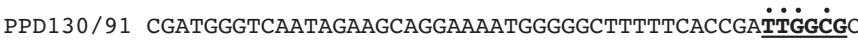
FK1051

E01-17 -

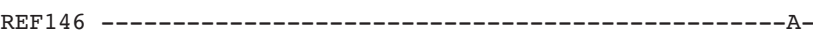

51

$-10$

100

PPD130/91 GAGGGTAAAAAAAATTAA $\dot{A} \dot{A} \ddot{A} \dot{\mathrm{T}} T$ TACTAAAGGTTGCCAAGGGGAGCGCC FK1051

E01-17

REF146

101

150

PPD130/91 GATACCTGAGGGGACGGTGGCTGATGACGCCGTAGGGCAATCAGGCCCGA FK1051

E01-17

REF146

151

S.D.

200

PPD130/91 ACGATTAACCGTGATGCGCAGTGGCGCAACATTCGA $\dot{\mathrm{A} \dot{G} \dot{G} \dot{A} A A G C A C A C T A}$ FK1051 -----------------------------------------------------

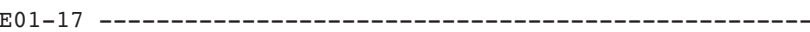
REF146

201

250

PPD130/91 TGGCACAAGTAATTAATACCAACAGCCTGTCGCTGATGGCACAGAACAAC FK1051

E01-17 REF146

251

300

PPD130/91 CTGAACAAATCCCAGTCAGCGCTGGGCACCGCCATCGAGCGTCTGTCTTC FK1051

E01-17 -----T-

REF 146 -----T-

301

350

PPD130/91 CGGTCTGCGCATCAACAGCGCCAAGGATGACGCCGCCGGTCAGGCGATCT FK1051

E01-17

REF146

351

400

PPD130/91 CCAACCGCTTCACTGCCAACATCAACGGCCTGACCCAGGCATCTCGCAAC FK1051 --------------C-----T---------------------C--------E01-17 --------------C------------------------------C-----REF146 ---------------C--------------------------------C------

401

PPD130/91 GCCAACGACGGTATCTCCCTGGCGCAGACCACCGAAGGCGCGCTGAACGA FK1051 --------------------------------------------------E01-17 -------------------------------------------------------

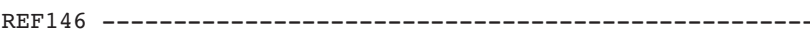
451

500

PPD130/91 AGTCAACGACAACCTGCAGAACATCCGTCGTCTGACCGTACAGGCACAGA

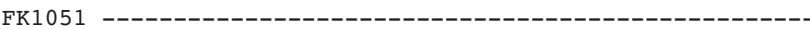
E01-17 ----------------------------T-----------G---T-----------

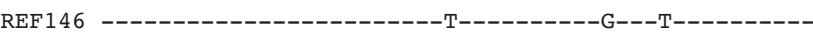

501

550

PPD130/91 ACGGCTCTAACTCCTCCAGCGACCTGCAGTCCATCCAGGACGAAATCACT FK1051 ------------------------------------------------------C E01-17 -------C--------------------------------------------C

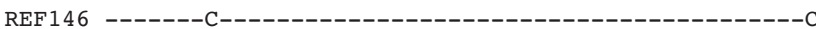

551

600

PPD130/91 CAGCGTCTGTCCGAGATCGACCGTATCTCCCAGCAGACCGACTTCAACGG

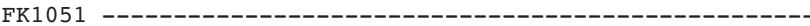
E01-17 -----------T------------------T--------------T-----

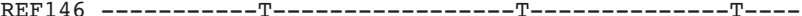

601

650

PPD130/91 CGTGAAGGTGCTGAGCAAAGATCAGAAGCTGACCATCCAGGTTGGCGCCA FK1051

E01-17 T------------------G------------------T-----C--T--REF146 T-----------------G------------------T-----C--T----

651

700

PPD130/91 ACGACGGTGAAACCATCGATATCGATCTGAAAAACATCAACGCACAGAGC

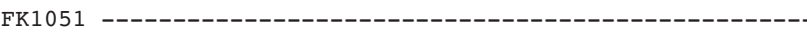
E01-17 ------------------------------------T---------------

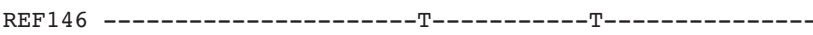
701

PPD130/91 CTGGGCCTGGATAAGTTTAACGTGGCTGACAGCGTTGACACGACTAAGGI FK1051

E01-17

REF 146

751

800

PPD130/91 TGCCGCTGCCGCTCCGGCTAAAGTGGAAACCAATATCGATGTTGCTATTA FK1051 ----------------------------A-----------------------E01-17 ------------------G------A-G------G-T-----------CREF146 --------------------G-------A-G-------G-T------------C801

850

PPD130/91 ACAATGATGCGACATTAAAAGCTGACTCTAAAGACATTACTGGTTATGAG FK1051 -----------------------------------------------C--E01-17 C---C---C-G----G--------G-GG-------T---G-CA-C-----REF146 C---C---C--G----G--------G-GG------T---G-CA-C-----851

900

PPD130/91 CAGAAAGGTGCTGACCTGTATGCGAAAAACACCGATGGTAAGCTGTTTAA FK1051 -----G--------------------------------------------E01-17 -----G----A---T----------------TG-------C-----------REF 146 -----G----A---T---------------TG-------C----------901

PPD130/91 AGTAACTACTATCGATAACACTACAGGTAAAGTTACTGGCGTTGATACTA FK1051 ----------------------------------------------E01-17 -----------------C------C-------CG--G-C-A-----G--REF146 -----------------CC------C--------CG--G-C-A-----G---

PPD130/91 CCGAATATACCGGTGGCGGTACTACAGCCGTAACCTCTATCAAGAAAGAA

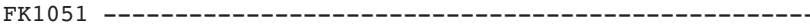
E01-17 -A--G--C----C---T---------------------------------

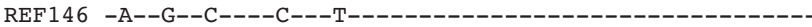
1001

1050

PPD130/91 GTTGCCCCGACCGGTCCGGATGCAGCTAATCTTCGTGCATATAGCGGTAC FK1051 --------------------------------------------------E01-17 ----------------C------A-C-------G-----------C-C-----REF146 --------------------A-A-C------G----------C-C----1051

1100

PPD130/91 TGAAAAAGGCGCTTCCGCCTATGTTATTCAGGAAGGTACCGGTGCTGACG

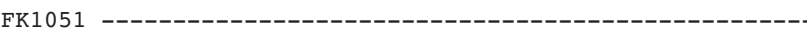
E01-17 C------G--------------------------------C-T--C------REF146 C-----G------------------------------C--T--C------1101

1150

PPD130/91 CTAAATACTTTAAGGCTAGCGTTGCCGATGATGGCACCGTGACCAAAGGC FK1051 -----------------------------------------------------

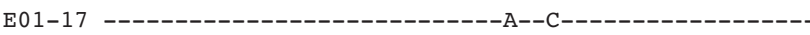

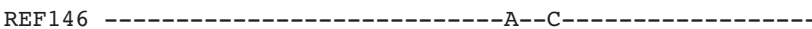

1151

1200

PPD130/91 TCTGCCCTGTCTACCACTGTTAAGACCGCCGATCCGCTGGCAACCCTGGA FK1051 ------------------C---------------------------------

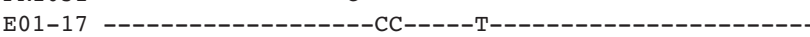
REF146 ---------------------CC------T-

(Fig. 2 continued on next page) 
1201

PPD130/91 TAAAGCCCTGTCTCAGGTTGATGACCTGCGCAGCGGCCTGGGTGCGGTAC
FK1051 $10 .-1300$
E01-17

PPD130/91 AGAACCGTTTCGATTCCGTTATCAACAACCTGAACAGCACCGTGAACAAC FK1051

E01-17

REF 146

1301

1350

PPD130/91 CTGTCCGCTTCCCGTTCACGTATTCAGGACGCTGACTACGCGACCGAAGT

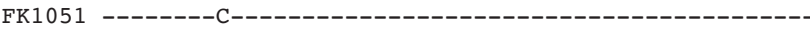

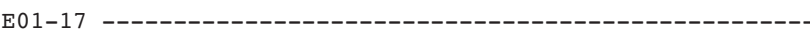

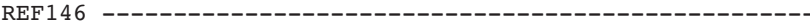

1351

1400

PPD130/91 GTCCAACATGAGCCGTGCGCAGATCCTGCAGCAGGCCGGTACCTCCGTAC

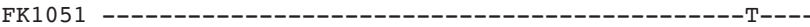

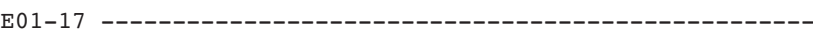
REF 146

1401

1450

PPD130/91 TGGCTCAGGCTAACCAGTCTACCCAGAACGTCCTGTCTCTGCTGCGTTAA FK1051 ----C--

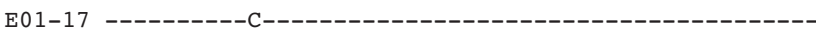

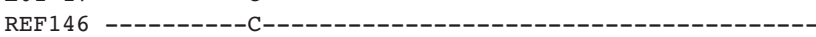

Fig. 2 (continued) sequence involved in the control of fliC transcription. The $199 \mathrm{bp}$ sequence located upstream of the fliC ORF contains the presumed promoter sequence (Fig. 2). This was amplified from FK1051, E01-17 and REF146 using PCR. The amplified PCR fragments were cloned and sequenced. Two bp differences were detected in the 199 bp upstream sequences of E01-17 and REF146 compared with that of FK1051 (Fig. 2). One base difference at nucleotide position 49 (guanine vs. adenine) was common to E01-17 and REF146. To examine the influence of these base differences on the transcription of the fliC gene, the ability of the 199 bp upstream sequence to initiate transcription of the downstream gene was compared. A lac $Z$ transcriptional fusion was constructed using a lacZ fusion vector plasmid as described in 'Materials and methods'. The $240 \mathrm{bp}$ DNA sequence comprising the $199 \mathrm{bp}$ sequence upstream of the fliC ORF and the $41 \mathrm{bp} 5^{\prime}$ proximal sequence of the fliC ORF was fused to lacZ. The $\beta$-galactosidase activities of the promoter-fusions in an E. coli background were compared. There was no significant difference in the ability of the $199 \mathrm{bp}$ upstream sequence to stimulate downstream transcription using either the motile strain or the non-motile strain's promoter regions (Table 3).

1

40

80

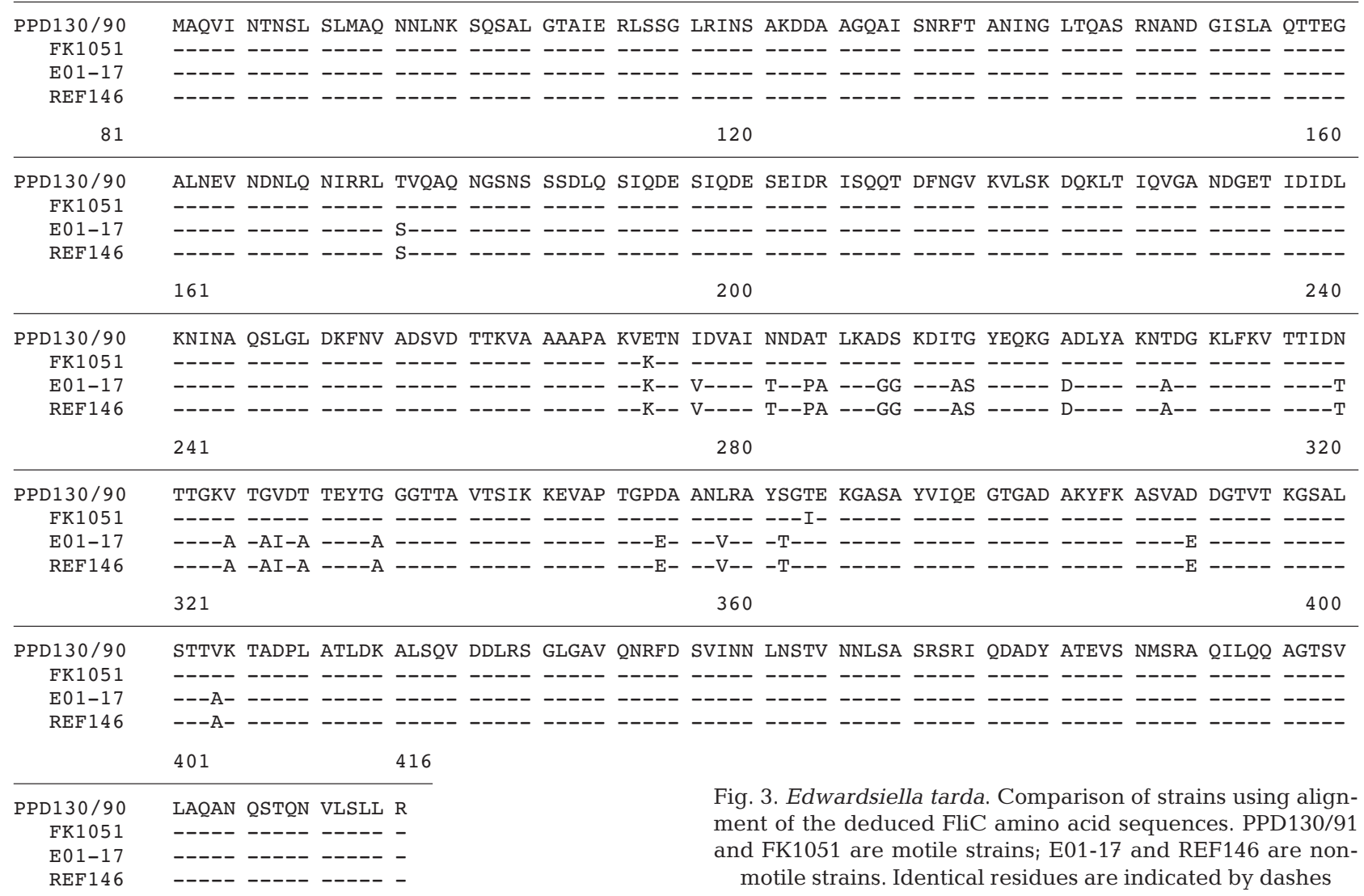




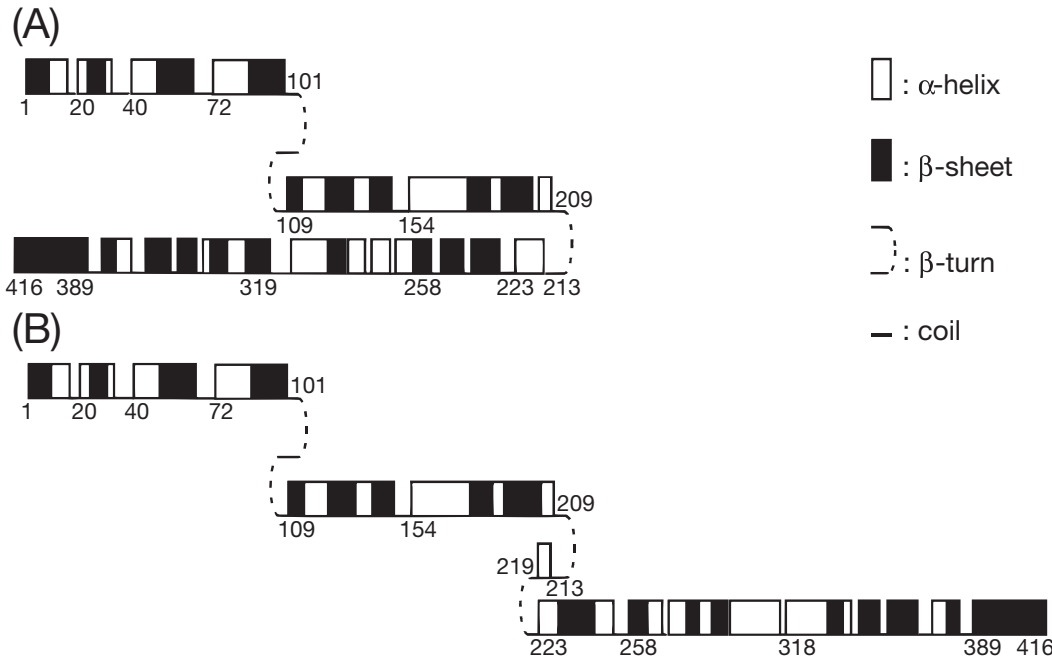

Fig. 4. Edwardsiella tarda. Predicted secondary structure of FliC from the deduced amino acid sequence. Secondary structure prediction was performed using the Chou-Fasman algorithm (Chou \& Fasman 1978). (A) Motile strain FK1051.

(B) Non-motile strains E01-17 and REF146

Table 3. $\beta$-galactosidase activities of E. coli DH5 $\alpha$ derivatives carrying fliC-lac $Z$ transcriptional fusions. Values are mean \pm SD of 3 independent experiments

\begin{tabular}{|cc|}
\hline $\begin{array}{c}\text { Origin (strain) } \\
\text { of the fliC gene }\end{array}$ & $\begin{array}{c}\beta \text {-galactosidase } \\
\text { activity (units) }\end{array}$ \\
\hline FK1051 & $2174 \pm 622$ \\
E01-17 & $2247 \pm 216$ \\
REF146 & $2051 \pm 375$ \\
\hline
\end{tabular}

Furthermore, we examined whether the fliC gene is actually transcribed to the same level in motile and non-motile strains by RT-PCR, as described in 'Materials and methods'. To rule out the possibility that reverse-transcribed cDNA contained genomic DNA, we also examined the amount of contaminating chromosomal DNA in each sample and found that there was no amplification in control reactions without reverse transcriptase (data not shown). As shown in Fig. 5, the fliC transcripts were detected in all Edwardsiella tarda strains examined. To perform relative quantification of the fliC transcript levels, the relative expression level of the flic transcript was measured by normalizing the PCR product of the fliC gene to that of the gyrA gene. The relative expression level of the fliC transcript was calculated as the ratio of the RT-PCR product of the fliC gene to that of the gyrA gene (the fliC: gyrA ratio) (Fig. 5). There was no significant difference in the relative fliC transcript levels (the fliC: $g y r A$ ratio) between motile and non-motile strains: the fliC: gyrA ratios ranged from 0.8 to 1.5 in the 7 motile strains and from 0.9 to 1.4 in the 6 non-motile strains (Fig. 5). These results confirmed that the fliC gene is actually transcribed in motile and non-motile strains to the same level.

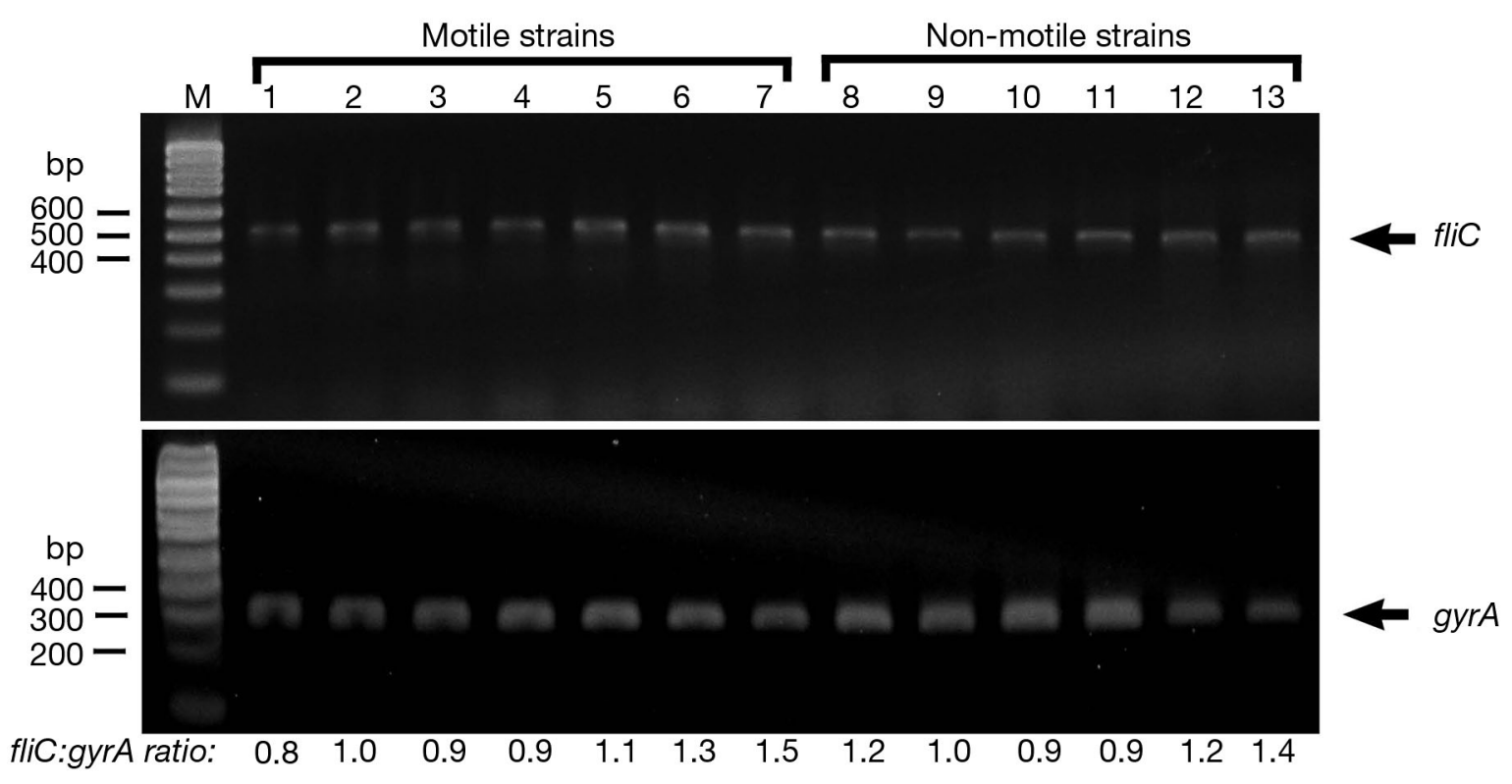

Fig. 5. Edwardsiella tarda. RT-PCR analysis of the fliC transcript levels. Lanes: M, 100 bp DNA ladder marker; 1 , FK1051; 2, NUF251; 3, NUF82; 4, PE113; 5, E01-33; 6, E01-37; 7, NE8003; 8, E01-14; 9, E01-17; 10, E01-40; 11, REF179; 12, REF146; 13, REF0001. The gyrA gene was used as an internal control. The relative expression level of the fliC transcript was calculated as the ratio of the RT-PCR product of the fliC gene to that of the gyrA gene (fliC: gyrA ratio) 


\section{DISCUSSION}

Bergey's manual (Sakazaki 2001) describes 2 types of Edwardsiella tarda, whereby most strains are motile with peritrichous flagella and produce no acids from L-arabinose, sucrose, trehalose, or D-mannitol; in comparison, E. tarda strains belonging to Biogroup 1 (Grimont et al. 1980) are also motile but produce acids from L-arabinose, sucrose, and D-mannitol. The E. tarda strains isolated for this study from Japanese flounder all showed the same characteristics as the former strains in Bergey's manual. The atypical isolates from red sea bream were differentiated from the typical strains by the lack of motility and the ability to utilize L-arabinose only; however, the previously reported non-motile E. tarda strains produced acid from D-mannitol as well as from L-arabinose (Kusuda et al. 1977, Yasunaga et al. 1982). There is no specific description of non-motile E. tarda strains in Bergey's manual. Our results suggest it may be appropriate to classify E. tarda into 3 biogroups, with the non-motile strains belonging to a new biogroup.

Motility was detected only in Edwardsiella tarda strains isolated from Japanese flounder, not in those isolated from red sea bream, and motility was associated with flagella formation (Table 1). Unlike Aeromonas salmonicida, motility and flagella formation were not influenced by the incubation temperature. The possibility that an occurrence of fliC ORF disruption by nonsense mutation or frame-shift mutation in non-motile strains was ruled out because full-length FliCs were encoded in the fliC ORFs of non-motile strains. However, amino acid residue substitutions at 22 positions were detected in the predicted FliC amino acid sequence of the non-motile strains (Fig. 3). Some of these substitutions significantly influenced the secondary structure of FliC in computer generated models (Fig. 4, discussed below). We next examined the possibility of mutations in the $199 \mathrm{bp}$ upstream sequences of the fliC ORF containing the presumed fliC promoter. The sequence and lac $Z$ fusion analyses suggested that FliC would be produced in non-motile strains at similar levels as in motile strains unless some other unknown factors are involved in fliC expression control. We then examined if the fliC gene is actually transcribed by RTPCR in motile and non-motile strains to the same level. As shown in Fig. 5, the fliC transcripts were detected in all E. tarda strains examined, and there was no significant difference in the relative fliC transcript levels (fliC:gyrA ratio) between motile and non-motile strains.

We therefore consider that an alteration in the secondary structure of FliC in non-motile strains may be an important factor responsible for non-flagellation in non-motile strains. The hairpin loop structure pre- dicted for FliC in the motile strain FK1051 was disrupted in non-motile strains E01-17 and REF146 owing to the additional $\beta$-turn, and an extended form was predicted instead for the secondary structure (Fig. 4). The $\mathrm{N}$ - and C-terminal conserved domains of flagellin (the D0 and D1 domains) form packed $\alpha$-helix structures, whereby the packed $\alpha$-helix regions are embedded in the flagellum inner core and are essential for filament architecture and motility functions (Ramos et al. 2004). In FK1051, the $\mathrm{N}$ - and C-terminal domains of flagellin (the D0 and D1 domains) form packed $\alpha$-helix structures owing to the hairpin loop structure of FliC. Because of the disruption of the hairpin loop structure in non-motile strains, the $\mathrm{N}$ - and $\mathrm{C}$-terminal domains would not form packed $\alpha$-helix structures, and may result in a failure in the construction of the filament architecture. Another possible explanation is failure to transport a defective form of FliC in non-motile strains. FliC is synthesized in the cytoplasm and exported through the central channel by the flagellum-specific export apparatus to the site of assembly at the distal end of the growing filament (MacNab 2004). Non-flagellated strain FliC might not be transported through the central channel because of the drastic alteration in the secondary structure in non-motile strains. These possibilities need to be studied in the future.

In this study, we speculate about the possible role of a modified FliC in the non-motile behavior of certain Edwardsiella tarda strains. However, there are several dozen proteins involved in the export and assembly of flagella and the motor-export complex, and mutations in any of their corresponding genes may result in nonfunctional motility and lack of flagellin export. Transformation of FliC knockout bacterial models with the non-motile E. tarda FliC gene would help clarify its effect on motility and flagella formation.

Acknowledgements. This research was partially supported by a Grant-in-Aid for Scientific Research (No. 18580184) from the Ministry of Education, Science, Sports and Culture of Japan, and by a Grant-in-Aid for Scientific Research (No. 18075) from the Ministry of Agriculture, Forestry and Fisheries of Japan.

\section{LITERATURE CITED}

Chou PY, Fasman GD (1978) Prediction of the secondary structure of proteins from their amono acid sequence. Adv Enzymol 47:45-147

Clarridge JE, Musher DM, Fainstein V, Wallace RJ (1980) Extraintestinal human infection caused by Edwardsiella tarda. J Clin Microbiol 11:511-514

Cook RA, Tappe JP (1985) Chronic enteritis associated with Edwardsiella tarda infection in Rockhopper penguins. J Am Vet Med Assoc 187:1219-1220

de Lorenzo V, Herrero M, Jakubzik U, Timmis KN (1990) Mini-Tn5 transposon derivatives for insertion mutagenesis, promoter probing, and chromosomal insertion of 
cloned DNA in gram-negative eubacteria. J Bacteriol 172: 6568-6572

Goldstein EJC, Agyare EO, Vagvolgi AE, Halpern M (1981) Aerobic bacterial oral flora of garter snakes: development of normal flora and pathogenic potential for snakes and humans. J Clin Microbiol 13:954-956

Grimont PAD, Grimont F, Richard C, Sakazaki R (1980) Edwardsiella hoshinae, a new species of Enterobacteriaceae. Curr Microbiol 4:347-351

Hoshina T (1962) On a new bacterium, Paracolobactrum anguillimortiferum. Bull Jpn Soc Sci Fish 28:162-164

Janda JM, Abbott SL (1993) Infections associated with the genus Edwardsiella: the role of Edwardsiella tarda in human disease. Clin Infect Dis 17:742-748

Jordan GW, Hadley WK (1969) Human infection with Edwardsiella tarda. Ann Intern Med 70:283-288

Kourany M, Vasquez MA, Saenz R (1977) Edwardsiellosis in man and animals in Panama: clinical and epidemiological characteristics. Am J Trop Med Hyg 26:1183-1190

Kusuda R, Itami T, Munekiyo M, Nakajima H (1977) Characteristics of a Edwardsiella sp. from an epizootic of cultured crimson sea breams. Bull Jpn Soc Sci Fish 43:129-134

MacNab RM (2004) Type III flagellar protein export and flagellar assembly. Biochim Biophys Acta 1694:207-217

Matsuyama T, Kamaishi T, Ooseko N, Kurohara K, Iida T (2005) Pathogenicity of motile and non-motile Edwardsiella tarda to some marine fish. Fish Pathol 40:133-135

McIntosh D, Austin B (1991) Atypical characteristics of the salmonid pathogen Aeromonas salmonicida. J Gen Microbiol 137:1341-1343

Meyer FP, Bullock GL (1973) Edwardsiella tarda, a new pathogen of catfish (Ictalurus punctatus). Appl Microbiol 25: 155-156

Mowbray EE, Buck G, Humbaugh KE, Marshall GS (2003) Maternal colonization and neonatal sepsis caused by Edwardsiella tarda. Pediatrics 111:296-298

Muroga K (2001) Viral and bacterial diseases of marine fish and shellfish in Japanese hatcheries. Aquaculture 202:23-44

Nakatsugawa T (1983) Edwardsiella tarda isolated from cultured young flounder. Fish Pathol 18:99-101

Okuda J, Nishibuchi M (1998) Manifestation of the Kanagawa phenomenon, the virulence-associated phenotype, of Vibrio parahaemolyticus depends on a particular single base change in the promoter of the thermostable direct haemolysin gene. Mol Microbiol 30:499-511

Editorial responsibility: Catherine Collins,

Aberdeen, UK
Okuda J, Toyotome T, Kataoka N, Ohno M and 5 others (2005) Shigella effector IpaH9.8 binds to a splicing factor U2AF(35) to modulate host immune responses. Biochem Biophys Res Commun 333:531-539

Otis VS, Behler JL (1973) The occurrence of Salmonellae and Edwardsiella in the turtles of the New York Zoological park. J Wildl Dis 9:4-6

Plumb JA (1999) Edwardsiella septicaemias. In: Woo PTK, Bruno DW (eds) Fish diseases and disorders. CABI Publishing, London, p 479-521

Ramos HC, Rumbo M, Sirard JC (2004) Bacterial flagellins: mediators of pathogenicity and host immune responses in mucosa. Trends Microbiol 12:509-517

Sakazaki R (2001) Genus XI. Edwarsiella Ewing and McWhorter 1965, 37 ${ }^{\mathrm{AL}}$. In: Brenner DJ, Krieg NR, Staley JT, Garrity GM (eds) Bergey's manual of systematic bacteriology, 2nd edn, Vol 2. Springer, New York, p 657-661

Sharma VK, Kaura YK, Singh IP (1974) Frogs as carriers of Salmonella and Edwardsiella. Antonie Leeuwenhoek 40: 171-175

Shitara M (1988) Flagellar stain. In: Senshokuho no subete, Medical Technology. Ishiyaku Publishers, Tokyo, p 299-301

Tan YP, Lin Q, Wang XH, Joshi S, Hew CL, Leung KY (2002) Comparative proteomic analysis of extracellular proteins of Edwardsiella tarda. Infect Immun 70:6475-6480

Tanaka M, Hasegawa T, Okamoto A, Torii K, Ohta M (2005) Effect of antibiotics on group A streptococcus exoprotein production analyzed by two-dimensional gel electrophoresis. Antimicrob Agents Chemother 49:88-96

Van Assche J (1991) Edwardsiella tarda infection in a puppy with possible parvovirus infection. Vet Rec 129:475-476

Wakabayashi H, Egusa S (1973) Edwardsiella tarda (Paracolobactrum anguillimortiferum) associated with pondcultured eel disease. Bull Jpn Soc Sci Fish 39:931-936

Wilson J, Waterer R (1989) Serious infections with Edwardsiella tarda. A case report and review of the literature. Arch Int Med 149:208-210

Winsor DK, Bloebaum AP, Mathewson JJ (1981) Gramnegative, aerobic, enteric pathogens among intestinal microflora of wild turkey vultures (Cathartes aura) in west central Texas. Appl Environ Microbiol 42:1123-1124

Yasunaga N, Ogawa S, Hatai K (1982) Characteristics of the fish pathogen Edwardsiella isolated from several species of cultured marine fishes. Bull Nagasaki Prefect Inst Fish 8: 67-73

Submitted: November 6, 2006; Accepted: March 29, 2007

Proofs received from author(s): May 28, 2007 